\title{
Tenascin Expression at the Invasive Front Is Associated with Poor Prognosis in Intrahepatic Cholangiocarcinoma
}

\author{
Shin-ichi Aishima, M.D., Ken-ichi Taguchi, M.D., Takahiro Terashi, M.D., Shuji Matsuura, M.D., \\ Mitsuo Shimada, M.D., Masazumi Tsuneyoshi, M.D. \\ Department of Anatomic Pathology (SA, TT, SM, MT) and Department of Surgery and Sciences (MS), \\ Graduate School of Medical Sciences, Kyushu University; and Kyushu National Cancer Center (KT), \\ Fukuoka, Japan
}

Tenascin and decorin are components of the extracellular matrix (ECM) that are implicated in cell proliferation in tumors. Here, we propose that abnormal expression of stromal ECM may play an important role in the progression of intrahepatic cholangiocarcinoma, which is characterized by desmoplastic reaction. To explore this hypothesis, we performed immunohistochemical analysis in order to examine the expression and distribution of tenascin and decorin in 75 cases of intrahepatic cholangiocarcinoma. In the intratumoral stroma, positive staining for tenascin was observed in $51(68 \%)$ cases, and positive staining for decorin was observed in $61(81 \%)$ cases. However, at the invasive front, positive staining for tenascin was found in $\mathbf{2 3}$ (31\%) cases, and positive staining for decorin was found in $6(8 \%)$ cases. Decorin staining was not correlated with aggressive behavior of intrahepatic cholangiocarcinoma, whereas intratumoral tenascin staining was correlated with lymphatic permeation and proliferative activity measured by Ki67. Tenascin staining at the invasive front was associated with tumor size, lymphatic permeation, lymph node metastasis, and proliferative activity and appeared to be a useful prognostic factor by univariate analysis, although it was not an independent prognostic factor. These results indicate that tenascin plays a role in tumor progression in cases of intrahepatic cholangiocarcinoma and that tenascin expression, especially at the invasive front, may be a useful marker in evaluating an unfavorable prognosis in patients with intrahepatic cholangiocarcinoma.

Copyright (C) 2003 by The United States and Canadian Academy of Pathology, Inc

VOL. 16, NO. 10, P. 1019, 2003 Printed in the U.S.A.

Date of acceptance: June 16, 2003.

Address reprint requests to: Masazumi Tsuneyoshi, M.D., Department of Anatomic Pathology, Graduate School of Medical Sciences, Kyushu University, 3-1-1 Maidashi, Higashi-ku, Fukuoka 812-8582, Japan; fax: 81-92642-5968; e-mail: masazumi@surgpath.med.kyushu-u.ac.jp.

DOI: $10.1097 / 01 . M P .0000086860 .65672 .73$
KEY WORDS: Decorin, Intrahepatic cholangiocarcinoma, Invasive front, Prognosis, Tenascin.

Mod Pathol 2003;16(10):1019-1027

Tenascin, a high molecular weight glycoprotein of the extracellular matrix (ECM), is involved in fetal development and oncogenesis (1). It is slightly expressed in a few types of normal adult tissue and is strongly expressed in many types of neoplastic tissue (2). Epidermal growth factor (EGF)-like repeats of tenascin directly bind to EGF receptor (EGFR) and activate the EGFR kinase cascade, which mainly participates in high proliferative and migratory activity (3-5). Several studies have previously demonstrated that tenascin expression in malignant tumors was related to proliferative activity and to invasive and metastatic potential (6-10).

Decorin, a small leucine-rich proteoglycan with the core protein, has been implicated in the control of cell proliferation $(11,12)$. It is considered as a novel biologic ligand for EGFR, and interaction between decorin and EGFR might inhibit cell growth via up-regulation of p21 during tissue remodeling and cancer growth (13-17). Another important role of decorin, which is produced by TGF- $\beta$ stimulation, is to neutralize the activity of TGF- $\beta$ (18).

The prognosis of patients with intrahepatic cholangiocarcinoma is poor (19-21). Histologically, cholangiocarcinoma is frequently characterized by abundant stroma in the tumors. In a previous study, we categorized intrahepatic cholangiocarcinomas based on the degree of stromal reaction, and a scirrhous-type intrahepatic cholangiocarcinoma had a worse prognosis than a nonscirrhous one (22). However, little is known about the role of the stromal component of intrahepatic cholangiocarcinoma. Tumor cells have been considered to modify their microenvironment by changes in the tumorous stroma that facilitate invasion (23). Here, we propose that abnormal expression of stromal ECM may play an important role in the progression of intrahepatic cholangiocarcinoma. 
Immunohistochemical examination of breast cancer has shown that positive expression for tenascin at the invasion border was associated with aggressive tumor behavior $(7,8)$. Therefore, we investigated tenascin and decorin expression not only in the intratumoral stroma, but also at the invasive front. To clarify the potential role of tenascin and decorin in the progression of intrahepatic cholangiocarcinoma, we performed immunohistochemical analyses in order to examine the expression and distribution of tenascin and decorin in cases of intrahepatic cholangiocarcinoma. The results were then compared with the clinicopathological findings and survival rates.

\section{MATERIALS AND METHODS}

\section{Cases}

Seventy-five cases of surgically resected primary intrahepatic cholangiocarcinoma were obtained by the Department of Anatomic Pathology of Kyushu University between July 1987 and March 2001. The cases of absolute noncurative surgery and the patients who received preoperative chemotherapy were excluded because those factors may have affected their survival data. Based on the criteria of the Liver Cancer Study Group of Japan (24), we examined 75 cases of mass-forming types of intrahepatic cholangiocarcinoma. The cases studied were considered appropriate for the current investigation because the mass-forming type of intrahepatic cholangiocarcinoma showed a prominent stromal response and clearly formed the invasion border close to the noncancerous liver tissue. The patients consisted of 43 men and 32 women, with a median age of 64 (range, 33-90) years. Standard surgical treatment was performed in all of the cases, and lymph node metastasis occurred in 27 (36\%) of the 75 patients. The survival of the patients was evaluated by follow-up for a median period of 2.4 years. Measurement of overall survival rates started at the time of surgery.

The resected liver specimens were fixed in $10 \%$ formalin and were embedded in paraffin. All of the sections prepared from the largest cut surface of the tumor were reviewed, and they were stained routinely with hematoxylin and eosin for histological examination. Tumor size was recorded as the largest diameter in the fixed specimens. Seventy-five cases of intrahepatic cholangiocarcinoma were classified histopathologically into 2 papillary and 73 tubular adenocarcinomas (17 well differentiated, 29 moderately differentiated, and 27 poorly differentiated). Areas in which the largest cut surface revealed a larger volume of fibrous stroma than tumor cells were defined as scirrhous areas. Tumors having a scirrhous area of $>70 \%$, that is, those with a prominent stromal desmoplasia and a relatively low number of tumor cells, were defined as scirrhous-type intrahepatic cholangiocarcinomas (22).

\section{Immunohistochemistry}

Immunohistochemical staining was performed by the avidin-biotin-peroxidase complex technique. The primary antibodies used were a mouse monoclonal anti-human tenascin (TN2, Novocastra), diluted at 1:50; a mouse monoclonal antihuman decorin (115402, R\&D systems), diluted at 1:100; a mouse monoclonal anti-human EGFR (EGFR. 113, Novocastra), diluted at 1:100; and a mouse monoclonal antibody against Ki-67 antigen (MIB-1, Immunotech), diluted at 1:50. Although tenascin shares domains homologous to EGF, fibronectin, and fibrinogen, there is no crossreaction with these proteins. Sections for tenascin, EGFR, and Ki67 antigen were pretreated with citrate buffer (pH 6.0) for 15 minutes at $100{ }^{\circ} \mathrm{C}$ in a microwave oven. The sections for decorin were digested with $0.1 \%$ trypsin solution at $37{ }^{\circ} \mathrm{C}$ for 30 minutes. The slides were exposed to $10 \%$ nonimmunized serum in PBS for 10 minutes, and then the sections were incubated overnight at $4{ }^{\circ} \mathrm{C}$ with primary antibodies. Positive reaction was visualized with $\mathrm{H}_{2} \mathrm{O}_{2}$ containing 3,3'-diaminobenzidine tetrahydrochloride (3,3'-diaminobenzidine)-PBS, and the sections were counterstained with hematoxylin. We used invasive breast carcinoma as a positive control for tenascin and decorin staining. As a negative control, non-relevant mouse IgG was used in place of the specific antibodies at the equivalent dilutions.

\section{Evaluation of Immunostaining}

The immunostaining in the tissue was judged independently by three pathologists (S.A., K.T., and T.T.) who had no prior knowledge of the clinical data or other immunohistochemical results. The tumor nuclei, when stained brown, were considered to be positive for MIB-1. MIB-1-labeling index (LI) was determined by counting a total of 1000 tumor cells at high-power field. We evaluated the immunostaining in the entire tumor stroma and at the invasive front. The staining for tenascin and decorin in the stromal components within the entire tumor stroma was semiquantitatively classified into four groups as follows: - , when $<10 \%$ of the intratumoral stroma was positive; + , when $10 \%$ to $40 \%$ of the intratumoral stroma was positive; ++ , when $40 \%$ to $70 \%$ of the intratumoral stroma was positive; and +++ , when $>70 \%$ of the intratumoral stroma was positive. When $>10 \%$ of the intratumoral stroma showed positive staining, the case was positive. We defined the invasive front area as 
the tumor periphery close to the noncancerous liver tissue. Positive staining for tenascin and decorin at the invasive front was defined as follows: negative, when $<10 \%$ of the positive carcinoma cells or stromal components were present at the invasive front; positive, when $>10 \%$ of the positive carcinoma cells or stromal components were present at the invasive front. A cutoff value of $10 \%$ positive carcinoma cells was used for EGFR.

\section{Statistical Analysis}

The statistical results were assessed by means of the $\chi^{2}$ test, Fisher's exact probability test, and Mann-Whitney $U$ test. Continuous variables including MIB-1 LI and tumor size were divided into two groups according to the mean. Overall survival curves were estimated with the Kaplan-Meier method and were statistically analyzed with the Logrank (Mantel-Cox) test. The Cox proportional hazards model with a stepwise procedure was used in the multivariate survival analysis. A $P$ value of $<.05$ was considered to be statistically significant.

\section{RESULTS}

\section{Immunostaining for Tenascin and Decorin in Noncancerous Liver}

In the non-cancerous liver tissue, positive staining for tenascin was occasionally found in the sinusoids and in inflammatory areas. The stroma in the portal tracts and the mature fibrotic septa of the cirrhotic liver were negative (Fig. 1A). Decorin was observed as a faint extracellular deposit in the portal connective tissue of the noncancerous liver tissue. Results of positive staining increased parallel to the inflammatory activity, and strong staining was detected in the fibrotic septa of cirrhotic liver tissue (Fig. 1B).

Immunostaining for Tenascin and Decorin in the Intratumoral Stroma

Positive staining for tenascin in the intratumoral stroma was found in 51 (68\%) of the 75 cases of intrahepatic cholangiocarcinoma and was not only confined to the delicate stroma but also was observed in the desmoplastic stroma around the carcinoma cells, independent of the degree of stromal reaction (Fig. 1C-D). Sixty-one (81\%) of the 75 cases stained positive for decorin in the intratumoral stroma. The hyalinized sclerotic stroma around the carcinoma cells was strongly positive for decorin (Fig. 1E-F).

\section{Immunostaining for Tenascin and Decorin at the Invasive Front}

Twenty-three $(31 \%)$ of the 75 cases showed positive staining for tenascin at the invasive front, where positive staining for tenascin was detected mostly in the delicate stromal components located in the interstices of the cancer cell nests (Fig. 2A-B). We also observed tenascin-positive carcinoma cells at the invasive front and at the cancer-stromal interface (Fig. 2D). Positive staining for decorin in the invasive front was found in $6(8 \%)$ of the 75 cases. The delicate stroma located in interstices of cancer cell nests was negative for decorin (Fig. 2C). The results of immunostaining for tenascin and decorin are summarized in Table 1.

\section{EGFR Staining}

In the noncancerous liver parenchyma, weak staining for EGFR was found in the membrane of the hepatocytes. Positive staining for EGFR in the carcinoma cells was detected in 57 cases $(71 \%)$ and was localized mainly to the membrane and the cytoplasm of the carcinoma cells. Although the positive carcinoma cells were found throughout the entire tumor area, a comparison of EGFR and tenascin staining revealed a relatively similar distribution of both proteins in 18 of the 75 cases (Fig. 3A, B).

\section{Relationship of Tenascin and Decorin with Clinicopathologic Findings}

The results of the analysis of the relationship between tenascin immunostaining and the clinicopathological findings of the patients with intrahepatic cholangiocarcinoma are presented in Table 2. The MIB-1 LI in the carcinoma cells was distributed within a range of 0 and $65 \%$ (19.5). Tenascin staining at the invasive front was correlated with tumor size, lymphatic permeation, lymph node metastasis, and proliferative activity (MIB-1 LI). In addition, tenascin staining in the intratumoral stroma was correlated with lymphatic permeation and proliferative activity. Tenascin and decorin staining was not correlated with the extent of the stromal reaction. No correlation was detected between decorin staining and the clinicopathologic findings

\section{Overall Survival and Prognostic Factors}

In the univariate postoperative survival analysis, tumor size, lymphatic permeation, lymph node metastasis, and tenascin staining at the invasive front were significant prognostic factors. Figure 4 provides a comparison of the survival curves between positive and negative staining for tenascin. The prognosis of the patients with positive staining for tenascin at the invasive front was worse than that of the patients with negative staining. The multivariate analysis showed that the presence of lymphatic permeation, lymph node metastasis, and larger tumor size were independent prognostic factors (Ta- 

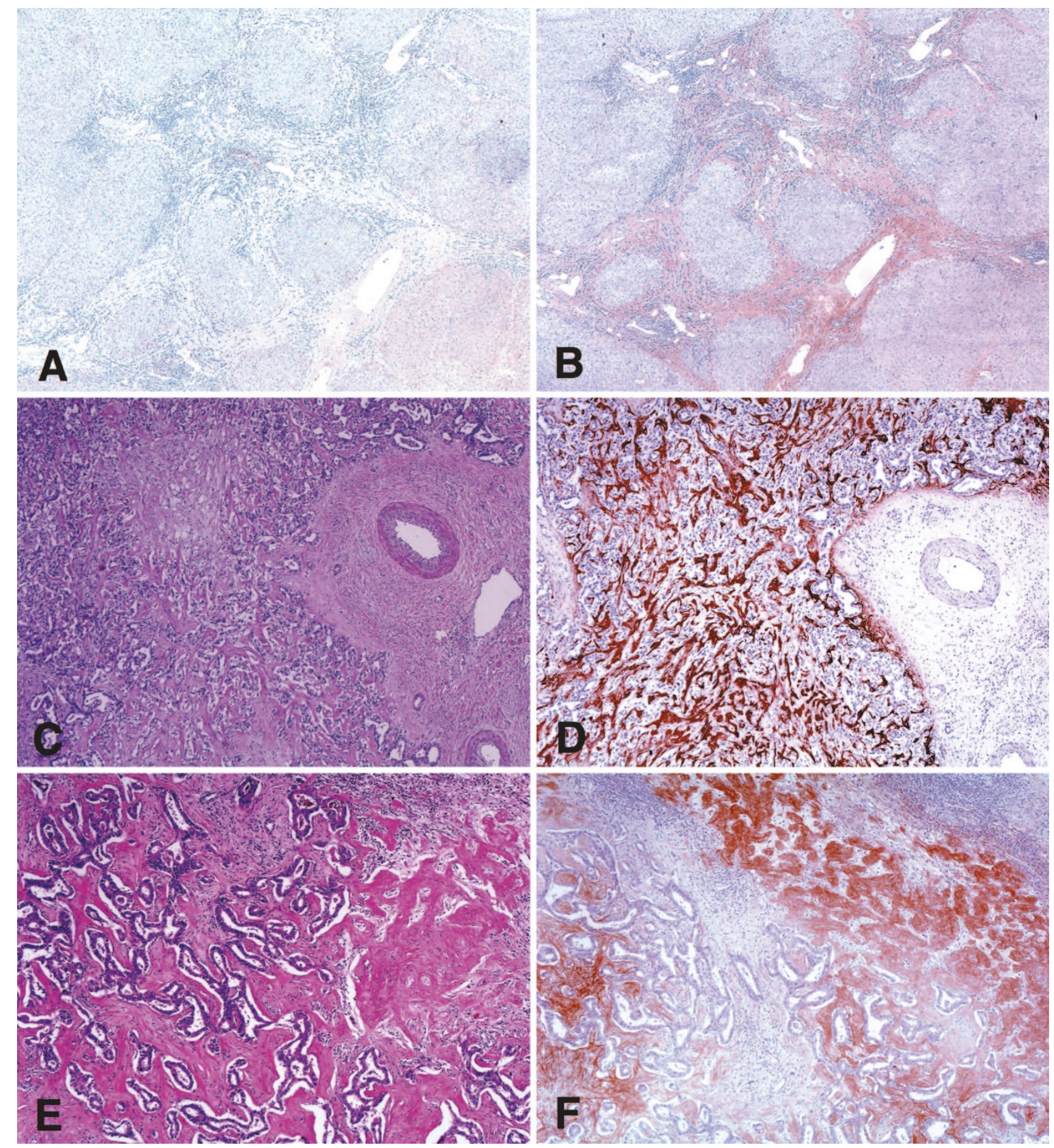

FIGURE 1. In the noncancerous cirrhotic liver, the mature fibrotic septa was negative for tenascin (A) but positive for decorin (B). Poorly differentiated adenocarcinoma proliferated with stromal desmoplasia (C). The stromal cells around the carcinoma cells were diffusely positive for tenascin, whereas the connective tissue in the involved portal tract was negative for tenascin (D). Moderately differentiated adenocarcinoma proliferated in a tubular fashion with hyalinized stroma (E). Mature hyalinized stroma showed strong decorin staining (F).

ble 3). Tenascin staining at the invasive front was not an independent prognostic factor.

\section{DISCUSSION}

It is known that diseased liver shows an increased accumulation of ECM, consisting of collagens, pro- teoglycans, and glycoproteins (2, 25-29). In intrahepatic cholangiocarcinomas, abnormal deposition of tenascin in tumor stroma has been reported, but not much is known regarding the correlation between tenascin expression, the clinicopathological features, and patient prognosis (30). We have found that positive staining for tenascin at the invasive 

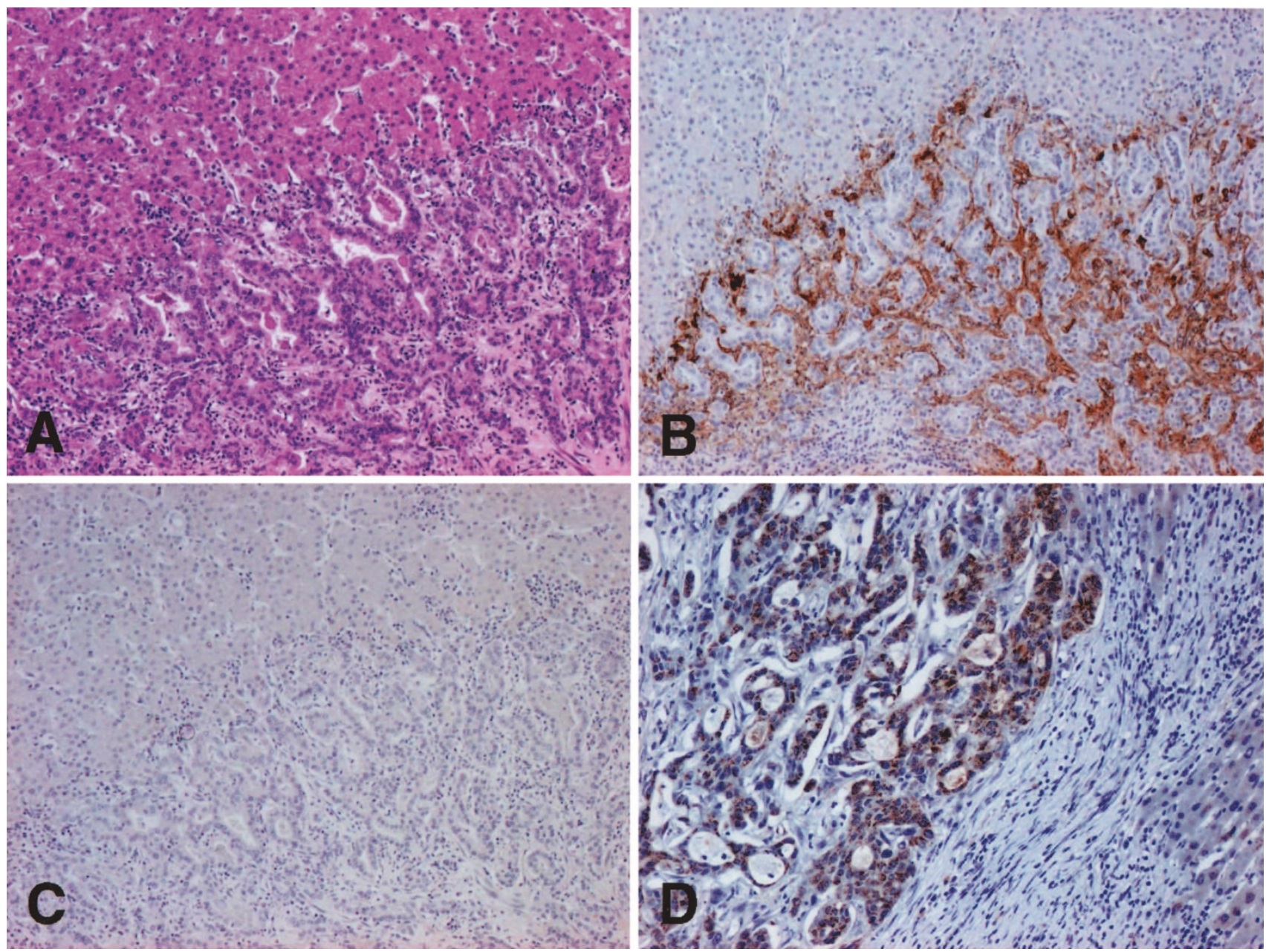

FIGURE 2. Moderately differentiated adenocarcinoma with delicate stroma invades to the liver parenchyma (A). At the invasive front, positive staining for tenascin was detected primarily in the delicate stromal components around the cancer cells (B); however, the delicate stroma was negative for decorin $(\mathbf{C})$. Tenascin staining was detected in the cytoplasm of carcinoma cells at the invasive front (D).

TABLE 1. Immunostaining for Tenascin and Decorin in Intrahepatic Cholangiocarcinomas

\begin{tabular}{|c|c|c|c|c|c|c|}
\hline & \multicolumn{6}{|c|}{ Immunostaining In: } \\
\hline & \multicolumn{5}{|c|}{ Intratumoral Stroma } & \multirow{3}{*}{ 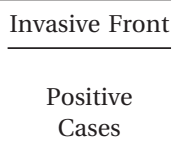 } \\
\hline & \multicolumn{4}{|c|}{ Staining Pattern } & \multirow{2}{*}{$\begin{array}{c}\text { Positive } \\
\text { Cases } \\
+\sim+++\end{array}$} & \\
\hline & - & + & ++ & $\overline{+++}$ & & \\
\hline Tenascin & 24 & 22 & 18 & 11 & $51(68 \%)$ & $23(31 \%)$ \\
\hline Decorin & 14 & 7 & 33 & 21 & 61 (81\%) & $6(8 \%)$ \\
\hline
\end{tabular}

front in cases with metastatic lymph nodes is higher than in those without metastatic lymph nodes. The findings suggest that tenascin expression at the invasive front was associated with a higher risk of lymph node metastasis. In this study, the possible utility of tenascin as a prognostic marker in cases of intrahepatic cholangiocarcinoma was examined. The survival rate of the patients with positive tenascin expression was worse than that of those patients with negative staining for tenascin. Thus, we identified tenascin as a significant prognostic marker, particularly when it was expressed at the invasive front. However, we have also shown that tenascin expression at the invasive front was significantly correlated with independent prognostic factors such as lymphatic permeation, lymph node metastasis, and tumor size. Therefore, tenascin expression at the invasive front of intrahepatic cholangiocarcinomas is not an independent prognostic marker.

Immunohistochemical analysis and in situ hybridization have led to the general conclusion that tenascin is synthesized not only by mesenchymal cells, but also by carcinoma cells $(9,26,31,32)$. Tenascin produced by carcinoma cells may be released promptly into the extracellular space, where it is then deposited. Some investigators have reported that tenascin expression in carcinoma cells was involved in tumor spreading and prognosis (6, 9). In the present study, the positivity of tenascin staining was higher in the intratumoral stroma than at the invasive front; however, we observed positive staining in the cytoplasm of cancer cells as well, 

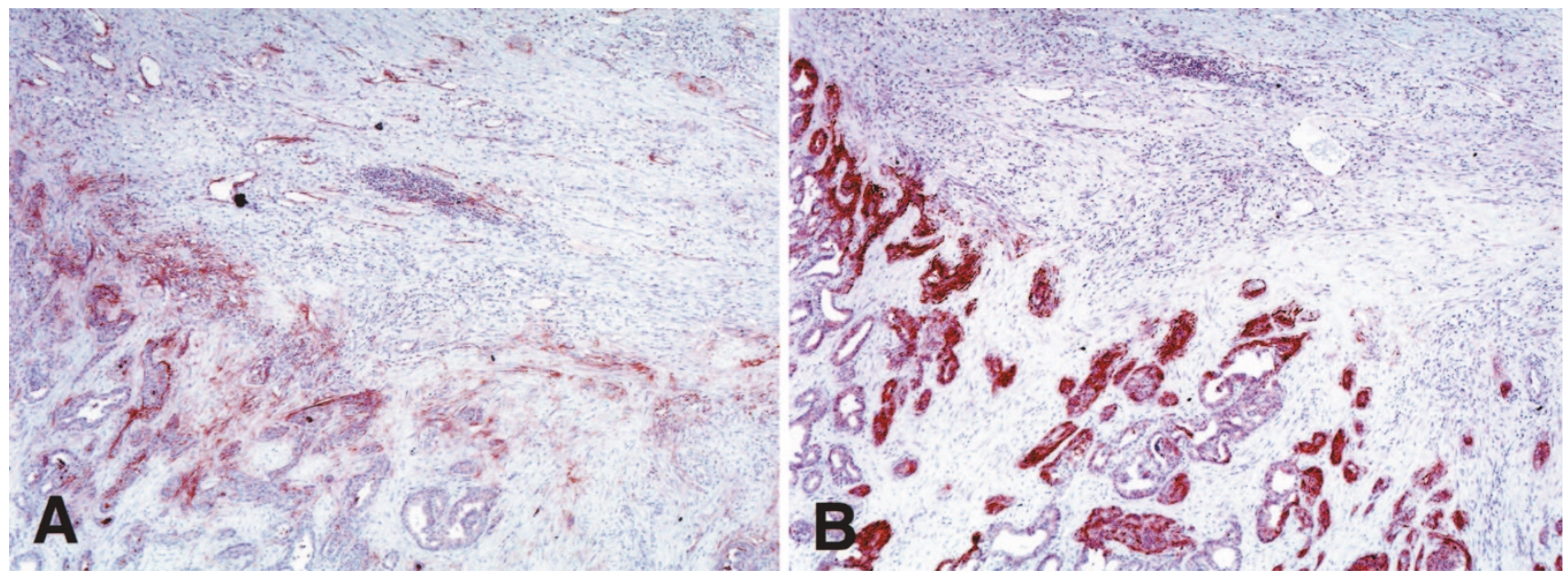

FIGURE 3. Tenascin staining was found in the stroma at the carcinoma-stroma interface (A). In the same tumor section, EGFR staining was detected in the membrane and cytoplasm of carcinoma cells at the invasive front (B).

TABLE 2. Comparison of Clinicopathological Findings for Tenascin Staining in Intrahepatic Cholangiocarcinomas

\begin{tabular}{|c|c|c|c|c|c|c|}
\hline \multirow{3}{*}{ Variable } & \multicolumn{3}{|c|}{ Intratumoral Stroma } & \multicolumn{3}{|c|}{ Invasive Front } \\
\hline & \multicolumn{2}{|c|}{ Tenascin } & \multirow[b]{2}{*}{$P$-Value } & \multicolumn{2}{|c|}{ Tenascin } & \multirow[b]{2}{*}{$P$-Value } \\
\hline & $(+)$ & $(-)$ & & $(+)$ & $(-)$ & \\
\hline Mean age (yr) & 65.2 & 62.3 & 0.2988 & 64.9 & 64.0 & 0.7542 \\
\hline Tumor size & & & 0.5070 & & & $0.0098^{*}$ \\
\hline$<4.5 \mathrm{~cm}$ & 24 & 14 & & 6 & 32 & \\
\hline$>4.5 \mathrm{~cm}$ & 27 & 10 & & 17 & 20 & \\
\hline Histological differentiation & & & 0.5061 & & & 0.4420 \\
\hline Well + Papillary & 11 & 8 & & 6 & 13 & \\
\hline Moderate & 20 & 9 & & 11 & 18 & \\
\hline Poor & 20 & 7 & & 6 & 21 & \\
\hline Stromal reaction & & & 0.7079 & & & 0.7168 \\
\hline Scirrhous & 12 & 4 & & 6 & 10 & \\
\hline Non-scirrhous & 39 & 20 & & 17 & 42 & \\
\hline Lymphatic permeation & & & $0.0494^{*}$ & & & $0.0198^{*}$ \\
\hline$(+)$ & 33 & 9 & & 18 & 24 & \\
\hline$(-)$ & 18 & 15 & & 5 & 28 & \\
\hline Venous invasion & & & 0.9110 & & & 0.0810 \\
\hline$(+)$ & 31 & 14 & & 17 & 28 & \\
\hline$(-)$ & 20 & 10 & & 6 & 24 & \\
\hline Lymph node metastasis & & & 0.1054 & & & $0.0277^{*}$ \\
\hline$(+)$ & 22 & 5 & & 13 & 14 & \\
\hline$(-)$ & 29 & 19 & & 10 & 38 & \\
\hline EGFR & & & 0.5381 & & & 0.3550 \\
\hline$(+)$ & 39 & 16 & & 19 & 36 & \\
\hline$(-)$ & 12 & 8 & & 4 & 16 & \\
\hline MIB-1 L.I. & & & $0.0082^{*}$ & & & $0.0098^{*}$ \\
\hline High & 31 & 6 & & 17 & 20 & \\
\hline Low & 20 & 18 & & 6 & 32 & \\
\hline
\end{tabular}

* Statistically significant.

especially at the invasive front. Tenascin expression in the intratumoral stroma was correlated with lymphatic permeation and proliferative activity, but expression at the invasive front was also correlated with lymph node metastasis and tumor size. According to the results, tenascin expression at the invasive front, some of which was possibly produced by the carcinoma cells, may promote tumor spreading. Tenascin expression at the invasive front of breast cancers has been considered to be related to aggressive tumor behavior $(7,8)$. We believe that tenascin expression in intrahepatic cholangiocarci- noma was more important as a prognostic marker at the invasive front than in the intratumoral stroma. Our results support the hypothesis that increased expression of tenascin at the invasive front induces the invasive potential of cancer cells. However, further experimental studies will be needed to clarify whether tenascin is capable of enhancing the malignant potential of cholangiocarcinoma cell lines.

We have found that tenascin was diffusely expressed in the tumor stroma and was also weakly detected in the sinusoids and in the inflamed 
Tenascin expression in intratumoral stroma

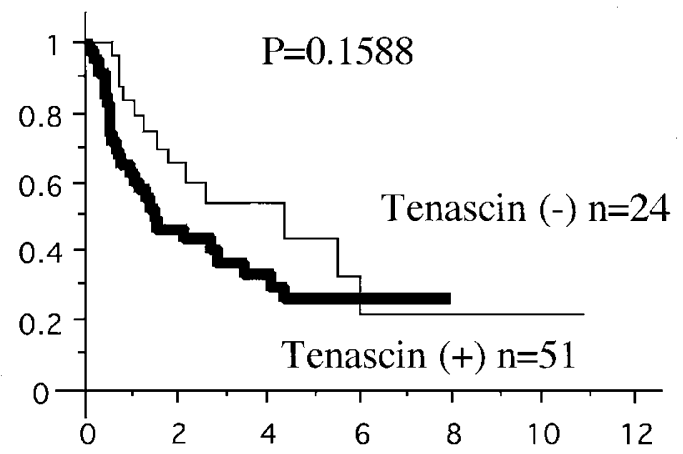

Years after surgery

Tenascin expression at the invasive front

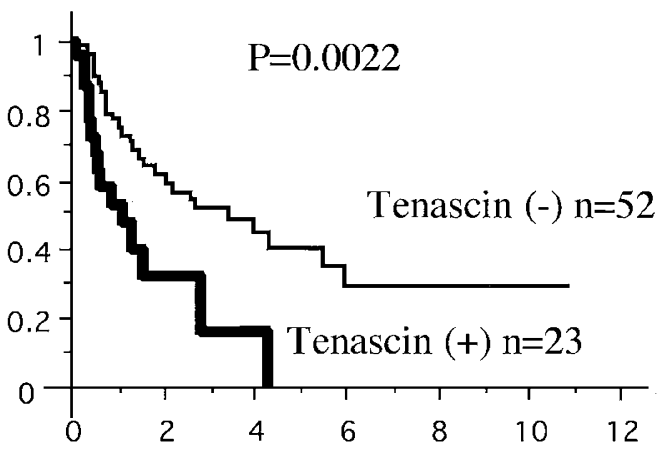

Years after surgery

FIGURE 4. Comparison of the survival curves between positive and negative staining for tenascin. The comparison of survival rates calculated by tenascin staining in the intratumoral stroma revealed no significant difference $(P=.1588$; A). However, the survival rate of the patients with tenascin-positive staining at the invasive front was shorter than that of patients with negative staining $(P=.0022 ; \mathbf{B})$.

TABLE 3. Multivariate Survival Analysis with Cox's Proportional Hazards Model

\begin{tabular}{lcccc}
\hline \multicolumn{1}{c}{ Variable } & Coefficient & Coef./S.E. & $\begin{array}{c}\text { Hazards } \\
\text { Ratio }\end{array}$ & $P$-Value \\
\hline Lymph node metastasis & 1.4592 & 3.8244 & 4.3027 & 0.0001 \\
Lymphatic permeation & 1.0417 & 2.7364 & 2.8340 & 0.0042 \\
Tumor size & 0.6892 & 2.0955 & 1.9920 & 0.0321 \\
\hline
\end{tabular}

S.E. $=$ standard error

stroma of the noncancerous liver; however, tenascin was rarely expressed in the mature fibrotic septa. Van Eyken et al. $(25,26)$ have demonstrated that the strong expression of tenascin in liver tissue was detected in the early septa, in contrast to its absence from the mature fibrous septa, thereby suggesting that tenascin plays a role in early matrix organization. Their results agree with immunohistochemical studies of various tissue types, including normal, hyperplastic, and neoplastic tissues (2). In the present study, tenascin expression was not

correlated to the extent of the stromal reaction. Our results, taken together with the previous findings, indicate that a tenascin-positive tumor stroma reflects an active remodeling matrix of intrahepatic cholangiocarcinoma, irrespective of the extent of the tumor stroma itself.

In contrast to tenascin, decorin is thought to suppress tumor cell growth and could be used as an effective anti-cancer agent (14-16, 33). Therefore, we analyzed the immunohistochemical staining results regarding two major ECM components, tenascin and decorin, in intrahepatic cholangiocarcinomas. Asakura et al. (34) reported dense staining for decorin in the mature central fibrotic area of pulmonary adenocarcinoma. There is evidence of increased expression of decorin during quiescence in mesenchymal cells (35). In the present study, decorin expression was detected in 61 (81\%) cases of tumor stroma, whereas decorin expression at the invasive front was only observed in 6 (8\%) cases. In addition, the mature stroma, that is, the intratumoral hyalinized area and fibrous septum of cirrhotic liver, had increased decorin expression. The results indicate that the intratumoral stroma of intrahepatic cholangiocarcinoma consists of mature fibrotic stroma. In contrast, mature and inactive stroma was rarely expressed at the invasive front of intrahepatic cholangiocarcinomas. Decorin expression was not correlated with parameters of aggressive tumor behavior. In the analysis of resected specimens of intrahepatic cholangiocarcinoma, we considered that decorin did not play a role in tumor suppression.

EGFR signaling was considered to play an important role in the growth and development of cancer cells; therefore, blockage of EGFR signaling would be important for anti-cancer therapies $(36,37)$. We also examined EGFR expression because both tenascin and decorin are ligands for EGFR. In 18 cases, EGFR and tenascin staining were detected in the same distribution in the tumor. The high level of proliferative activity in tenascin-positive intrahepatic cholangiocarcinomas may be attributed to a pathway mediated by EGFR that was stimulated by tenascin.

In conclusion, tenascin expressed at the invasive front may play an important role in enhancing the invasiveness of carcinoma cells and facilitating the metastatic potential of intrahepatic cholangiocarcinoma. This study provides new information regarding tenascin expression in the progression of intrahepatic cholangiocarcinoma. It was shown that tenascin expression at the invasive front of such cancers indicates an unfavorable prognosis for patients with intrahepatic cholangiocarcinoma. 


\section{REFERENCES}

1. Chiquet-Ehrismann R, Mackie EJ, Pearson CA, Sakakura T. Tenascin: an extracellular matrix protein involved in tissue interactions during fetal development and oncogenesis. Cell 1986;47:131-9.

2. Koukoulis GK, Gould VE, Bhattacharyya A, Gould JE, Howeedy AA, Virtanen I. Tenascin in normal, reactive, hyperplastic, and neoplastic tissues: biologic and pathologic implications. Hum Pathol 1991;22:636-43.

3. Swindle CS, Tran KT, Johnson TD, Banerjee P, Mayes AM, Griffith L, et al. Epidermal growth factor (EGF)-like repeats of human tenascin-C as ligands for EGF receptor. J Cell Biol 2001;154:459-68.

4. Jones FS, Burgoon MP, Hoffman S, Crossin KL, Cunningham BA, Edelman GM. A cDNA clone for cytotactin contains sequences similar to epidermal growth factor-like repeats and segments of fibronectin and fibrinogen. Proc Natl Acad Sci U S A 1988;85:2186-90.

5. Nies DE, Hemesath TJ, Kim JH, Gulcher JR, Stefansson K. The complete cDNA sequence of human hexabrachion (Tenascin). A multidomain protein containing unique epidermal growth factor repeats. J Biol Chem 1991;266:2818-23.

6. Ishihara A, Yoshida T, Tamaki H, Sakakura T. Tenascin expression in cancer cells and stroma of human breast cancer and its prognostic significance. Clin Cancer Res 1995;1:103541.

7. Jahkola T, Toivonen T, von Smitten K, Blomqvist C, Virtanen I. Expression of tenascin in invasion border of early breast cancer correlates with higher risk of distant metastasis. Int J Cancer 1996;69:445-7.

8. Jahkola T, Toivonen T, Virtanen I, von Smitten K, Nordling S, von Boguslawski $\mathrm{K}$, et al. Tenascin-C expression in invasion border of early breast cancer: a predictor of local and distant recurrence. Br J Cancer 1998;78:1507-13.

9. Yoshida T, Matsumoto E, Hanamura N, Kalembeyi I, Katsuta $\mathrm{K}$, Ishihara A, et al. Co-expression of tenascin and fibronectin in epithelial and stromal cells of benign lesions and ductal carcinomas in the human breast. J Pathol 1997;182: 421-8.

10. Kusagawa H, Onoda K, Namikawa S, Yada I, Okada A, Yoshida T, et al. Expression and degeneration of tenascin-C in human lung cancers. Br J Cancer 1998;77:98-102.

11. Krusius T, Ruoslahti E. Primary structure of an extracellular matrix proteoglycan core protein deduced from cloned cDNA. Proc Natl Acad Sci U S A 1986;83:7683-7.

12. Ruoslahti E, Yamaguchi Y. Proteoglycans as modulators of growth factor activities. Cell 1991;64:867-9.

13. Santra M, Skorski T, Calabretta B, Lattime EC, Iozzo RV. De novo decorin gene expression suppresses the malignant phenotype in human colon cancer cells. Proc Natl Acad Sci U S A 1995;92:7016-20.

14. Csordas G, Santra M, Reed CC, Eichstetter I, McQuillan DJ, Gross D, et al. Sustained down-regulation of the epidermal growth factor receptor by decorin. A mechanism for controlling tumor growth in vivo. J Biol Chem 2000;275:32879-87.

15. Iozzo RV, Moscatello DK, McQuillan DJ, Eichstetter I. Decorin is a biological ligand for the epidermal growth factor receptor. J Biol Chem 1999;274:4489-92.

16. Moscatello DK, Santra M, Mann DM, McQuillan DJ, Wong AJ, Iozzo RV. Decorin suppresses tumor cell growth by activating the epidermal growth factor receptor. J Clin Invest 1998;101:406-12.

17. De Luca A, Santra M, Baldi A, Giordano A, Iozzo RV. Decorin-induced growth suppression is associated with upregulation of $\mathrm{p} 21$, an inhibitor of cyclin-dependent kinases. J Biol Chem 1996;271:18961-5.

18. Border WA, Noble NA, Yamamoto T, Harper JR, Yamaguchi Y, Pierschbacher MD, et al. Natural inhibitor of transforming growth factor-beta protects against scarring in experimental kidney disease. Nature 1992;360:361-4.

19. Taguchi K, Aishima S, Asayama Y, Kajiyama K, Kinukawa N, Shimada M, et al. The role of p27kipl protein expression on the biological behavior of intrahepatic cholangiocarcinoma. Hepatology 2001;33:1118-23.

20. Sugimachi K, Aishima S, Taguchi K, Tanaka S, Shimada M, Kajiyama $\mathrm{K}$, et al. The role of overexpression and gene amplification of cyclin D1 in intrahepatic cholangiocarcinoma. J Hepatol 2001;35:74-9.

21. Aishima SI, Taguchi KI, Sugimachi K, Shimada M, Sugimachi K, Tsuneyoshi M. c-erbB-2 and c-Met expression relates to cholangiocarcinogenesis and progression of intrahepatic cholangiocarcinoma. Histopathology 2002;40:269-78.

22. Kajiyama K, Maeda T, Takenaka K, Sugimachi K, Tsuneyoshi $M$. The significance of stromal desmoplasia in intrahepatic cholangiocarcinoma: a special reference of "scirrhous-type" and "nonscirrhous-type" growth. Am J Surg Pathol 1999;23: 892-902.

23. Iozzo RV. Tumor stroma as a regulator of neoplastic behavior. Agonistic and antagonistic elements embedded in the same connective tissue. Lab Invest 1995;73:157-60.

24. Liver Cancer Study Group. The general rules for the clinical and pathological study of primary liver cancer. 4th ed. Tokyo: Kanehara Publications; 2000.

25. Van Eyken P, Geerts A, De Bleser P, Lazou JM, Vrijsen R, Sciot R, et al. Localization and cellular source of the extracellular matrix protein tenascin in normal and fibrotic rat liver. Hepatology 1992;15:909-16.

26. Van Eyken P, Sciot R, Desmet VJ. Expression of the novel extracellular matrix component tenascin in normal and diseased human liver. An immunohistochemical study. J Hepatol 1990;11:43-52.

27. Koukoulis GK, Shen J, Virtanen I, Gould VE. Vitronectin in the cirrhotic liver: an immunomarker of mature fibrosis. Hum Pathol 2001;32:1356-62.

28. Dudas J, Kovalszky I, Gallai M, Nagy JO, Schaff Z, Knittel T, et al. Expression of decorin, transforming growth factor-beta 1, tissue inhibitor metalloproteinase 1 and 2, and type IV collagenases in chronic hepatitis. Am J Clin Pathol 2001;115: 725-35.

29. Le Bail B, Faouzi S, Boussarie L, Balabaud C, Bioulac-Sage P, Rosenbaum J. Extracellular matrix composition and integrin expression in early hepatocarcinogenesis in human cirrhotic liver. J Pathol 1997;181:330-7.

30. Terada T, Nakanuma Y. Expression of tenascin, type IV collagen and laminin during human intrahepatic bile duct development and in intrahepatic cholangiocarcinoma. Histopathology 1994;25:143-50.

31. Zidar N, Gale N, Kambic V, Fischinger J. Expression of tenascin and fibronectin in benign epithelial hyperplastic lesions and squamous carcinoma of the larynx. Anticancer Res 2001; 21:451-4.

32. Tokes AM, Hortovanyi E, Csordas G, Kulka J, Mozes G, Hatalyak A, et al. Immunohistochemical localisation of tenascin in invasive ductal carcinoma of the breast. Anticancer Res 1999;19:175-9.

33. Santra M, Eichstetter I, Iozzo RV. An anti-oncogenic role for decorin. Down-regulation of ErbB2 leads to growth suppression and cytodifferentiation of mammary carcinoma cells. J Biol Chem 2000;275:35153-61.

34. Asakura S, Kato H, Fujino S, Konishi T, Tezuka N, Mori A. Role of transforming growth factor-betal and decorin in development of central fibrosis in pulmonary adenocarcinoma. Hum Pathol 1999;30:195-8.

35. Asundi VK, Dreher KL. Molecular characterization of vascular smooth muscle decorin: deduced core protein structure and regulation of gene expression. Eur J Cell Biol 1992;59: 314-32. 
36. Solorzano CC, Baker CH, Tsan R, Traxler P, Cohen P, Buchdunger E, et al. Optimization for the blockade of epidermal growth factor receptor signaling for therapy of human pancreatic carcinoma. Clin Cancer Res 2001;7: 2563-72.
37. Yoshida J, Ishibashi T, Nishio M. Growth-inhibitory effect of a streptococcal antitumor glycoprotein on human epidermoid carcinoma A431 cells: involvement of dephosphorylation of epidermal growth factor receptor. Cancer Res 2001; 61:6151-7. 